\title{
Efficacy and safety of Bailing capsules in the treatment of type 2 diabetic nephropathy: a meta-analysis
}

\author{
Xiaohua Sheng, Yang Dong, Dongsheng Cheng, Niansong Wang, Yongping Guo \\ Department of Nephrology, Affiliated Sixth People's Hospital, Shanghai Jiao Tong University, Shanghai, China \\ Contributions: (I) Conception and design: X Sheng, N Wang, Y Guo; (II) Administrative support: Y Dong; (III) Provision of study materials or \\ patients: X Sheng, Y Dong, D Cheng; (IV) Collection and assembly of data: All authors; (V) Data analysis and interpretation: X Sheng, D Cheng, N \\ Wang, Y Guo; (VI) Manuscript writing: All authors; (VII) Final approval of manuscript: All authors. \\ Correspondence to: Niansong Wang; Yongping Guo. Department of Nephrology, Affiliated Sixth People's Hospital, Shanghai Jiao Tong University, 600 \\ Yishan Road, Shanghai, China. Email: wangniansong2013@163.com; zhaoguoyongping@163.com.
}

\begin{abstract}
Background Diabetic nephropathy (DN) is the main cause of end-stage renal failure (ESRF) in diabetic patients. Chinese medicine plays an extremely important role in controlling the symptoms of DN. At present, the efficacy and safety of Bailing capsules in the treatment of type $2 \mathrm{DN}$ are still unclear. Therefore, the aim of this meta-analysis was to evaluate the clinical efficacy and safety of Bailing capsules in the treatment of type $2 \mathrm{DN}$.
\end{abstract}

Methods: A literature search on type 2 DN was conducted using Chinese and English databases. The Chinese databases searched were the CNKI database, Wanfang database, and Weipu database using the following search terms: Bailing capsule and DN. The English databases were PubMed, Embase, and Web of Science using the following search terms: type 2 diabetes mellitus, type II diabetes mellitus, and Bailing capsule. The quality of the literature was evaluated using RevMan 5.3 software. The meta-analysis was performed using the R3.5.1 software meta package.

Results: Twenty-four articles with a total of 985 patients in the treatment group and 956 patients in the control group were found. The total effective rate of Bailing capsules in the treatment group was 1.24 times that of the control group [95\% confidence interval (CI): 1.11-1.38]. Reductions in 24-h urine total protein, urine albumin excretion rate (UAER), serum creatinine (Scr), and blood urea nitrogen (BUN) levels before and after treatment in the treatment group were significantly lower than that of the control group, with standard mean differences (SMD) of 0.61 (95\% CI: -1.01 to -0.22 ), -1.56 (95\% CI: -2.34 to -0.78 ), -0.58 (95\% CI: -0.89 to -0.27 ), and -0.73 (95\% CI: -1.16 to -0.29 ), respectively. However, there was no significant change in serum potassium between the two groups $(\mathrm{P}>0.05)$. No publication bias was found in the metaanalysis $(\mathrm{P}>0.05)$.

Conclusions: For type 2 DN patients, the use of Bailing capsules in routine treatment demonstrated higher clinical efficacy and was found to improve the kidney function. However, high-quality randomized controlled trials are required to further explore the safety of Bailing capsules.

Keywords: Type 2 diabetic nephropathy (type 2 DN); Bailing capsule; meta-analysis

Submitted Aug 17, 2020. Accepted for publication Oct 24, 2020.

doi: 10.21037/apm-20-1799

View this article at: http://dx.doi.org/10.21037/apm-20-1799

\section{Introduction}

Diabetic nephropathy $(\mathrm{DN})$ is a microvascular complication of diabetes involving the glomerulus, renal tubules, and other renal structures, and is the main cause of end- stage renal failure (ESRF). With the incidence of diabetes increasing in recent years, the number of cases of DN has also increased. The National Diabetes Alliance estimates that, by 2030, there will be about 552 million people with 
diabetes globally, and nearly one-third of them will have DN (1). A survey in 2013 showed that the prevalence of type 2 diabetes was $10.4 \%$, and the incidence of DN was 20 $60 \%$ (2). The high incidence of $\mathrm{DN}$ and its high treatment cost are associated with heavy disease and economic burden, and seriously affect the quality of life of diabetic patients (3). The main clinical manifestations of $\mathrm{DN}$ are microproteinuria $[\geq 2$ times, $24 \mathrm{~h}$ urinary total protein (UTP) $>30 \mathrm{~g}$ ] for multiple occurrences, or large amounts of proteinuria ( $24 \mathrm{~h}$ urine protein $\geq 30 \mathrm{~g}$ ). Clinical treatment is mainly focused on controlling blood glucose level, reducing urine protein, and improving renal microcirculation (4). The treatment of DN in Western medicine often has longterm side-effects; therefore, in recent years, the role of Chinese medicine has been increasing and has been found to play an important role in controlling the symptoms of $\mathrm{DN}$ and delaying disease progression. Studies have found that traditional Chinese medicine has good curative effects in controlling blood glucose level, blood pressure, blood lipids, urine protein, serum creatinine ( $\mathrm{Scr}$ ), and blood urea nitrogen (BUN), which shows potential advantages in clinical practice with stable efficacy and low side-effects for relieving symptoms, such as back pain, edema, and fatigue (5).

The Bailing capsule is a medicine refined by lowtemperature fermentation of cordyceps strains that contains cordyceps polysaccharides and amino acids. It has various functions, such as antioxidative, anti-inflammatory, and proteinuria reduction (6-8), and can effectively treat DN. Meta-analyses have shown that the Bailing capsule, combined with Western medicine, has better efficacy than Western medicine alone to treat $\mathrm{DN}$ by reducing urine protein and protecting kidney function $(9,10)$. However, the efficacy and safety of Bailing capsules in the treatment of type $2 \mathrm{DN}$ are still unclear. Due to small sample sizes in studies of traditional Chinese medicine, non-standard randomized clinical trials (RCTs), and insufficient exploration of various empiric information in clinical data, there is a need to explore the clinical efficacy and safety analysis of Bailing capsules for type $2 \mathrm{DN}$ by analyzing the latest clinical evidence-based data. Therefore, in the present study, based on a randomized clinical controlled trial, we performed a meta-analysis of the clinical efficacy and safety of Bailing capsules, combined with the routine treatment of type $2 \mathrm{DN}$, for a more objective and comprehensive evaluation of this therapy to guide clinical application. We present the following article in accordance with the
PRISMA reporting checklist (available at http://dx.doi. org/10.21037/apm-20-1799).

\section{Methods}

\section{Search strategy}

A literature search on type $2 \mathrm{DN}$ was conducted using Chinese and English databases. The Chinese databases searched were the CNKI database, Wanfang database, and Weipu database using the following search terms: Bailing capsule and DN. The English databases were PubMed, Embase, and Web of Science using the following search terms: type 2 diabetes mellitus, type II diabetes mellitus, and Bailing capsule. The retrieval time was from the establishment of the database to May 20, 2020. Keywords of the same category of search terms were connected by "or", and drugs and diseases were connected by "and". To prevent omission of references in the included literature, Google Scholar was used for the searches.

\section{Literature inclusion and exclusion criteria}

The inclusion criteria were as follows: (I) the study participants were patients with type 2 diabetes and clinically diagnosed with DN (11); (II) the treatment measures for the control group were as follows: undergoing routine treatment, such as lower blood pressure, blood glucose level, and blood lipids levels, and/or combined with Western medicine for treatment; (III) patients in the experimental group were treated with Bailing capsule based on the treatment of the control group; (IV) the study design was RCTs; (V) the disease course and treatment course were not limited; and (VI) outcome indicators included efficacy and safety indicators; the first category of clinical efficacy indicators included the total treatment efficacy, the quantification of 24-h urine UTP, urine albumin excretion rate (UAER), Scr, and BUN; the second category included safety indicators, such as serum potassium and adverse reaction rate. The exclusion criteria were as follows: (I) reviews or animal experiment research; (II) conferences or case reports; (III) systematic reviews or meta-analyses; (IV) no research outcome indicators; and $(\mathrm{V})$ duplicate literature.

\section{Literature search and screening}

Two researchers conducted the literature search by independently reading and screening literature according 
to the same search criteria. They searched the literature in the databases and read the abstract or full text for screening. In case of disagreement, a third researcher made the final decision.

\section{Data extraction and literature quality evaluation}

A unified information collection form was used to extract data by two independent researchers. The extracted data included the following: (I) general characteristics, such as information of the first author, year of publication, control and experimental treatment methods, treatment sample size, and treatment time; (II) quality evaluation content, such as research type, random hiding method, blinding method, selective reporting, and other biases; and (III) outcome indicators of the study, such as the number of successfully treated cases and the means and standard deviations of 24-h UTP, UAER, Scr, BUN, and serum potassium levels before and after treatment.

Literature quality was evaluated according to the risk bias evaluation tools recommended by the Cochrane Group. The research type included the separate evaluation of random hiding method, blinding method, selective reporting, and other biases. The evaluation levels were divided into three levels: low risk, unclear, and high risk (12).

\section{Statistical methods}

The literature quality evaluation was evaluated using RevMan 5.3 software (The Cochrane Collaboration). The total rates of successfully treated cases were combined using the ratio risk and the $95 \%$ confidence interval (CI); levels of 24-h UTP, UAER, Scr, BUN, and serum potassium were combined using standard mean difference (SMD) and 95\% CI. Heterogeneity evaluation $\left(\mathrm{I}^{2}\right)$ was performed; when $\mathrm{I}^{2}$ $\leq 50 \%$, the fixed-effects model was used, and when $\mathrm{I}^{2}>50 \%$, the random-effects model was used to estimate efficacy. For heterogeneous studies, the sensitivity meta-analysis was performed after removing the literature that did not meet the inclusion criteria one by one. The funnel chart, Begg's rank correlation, and Egger's regression were used for the qualitative and quantitative evaluations of publication bias. The combination of effect values, forest maps, publication bias, sensitivity analysis, and other analyses of effect size, forest plot, publication bias, and sensitivity analysis were all statistically analyzed using the R3.5.1 software meta package.

\section{Results}

\section{Literature screening results}

A total of 732 articles were retrieved according to the search strategy, including 724 in Chinese and 8 in English. After removing conference reports, duplicates, animal experiments, reviews, systematic reviews, meta-analyses, articles that did not include type 2 diabetes patients, nonRCT studies, interventions not meeting the requirements, and literature with no outcome indicators, 24 articles were finally included. All included articles were in Chinese and involved 1,941 cases, including 985 in the test group and 956 in the control group. The retrieval flowchart is shown in Figure 1.

Details of the 24 included studies are shown in Table 1 . The sample size of the 24 studies was small. The control group measures included routine treatment and routine treatment combined treatment with Western medicine. The treatment course and the dosage of Bailing capsules in the experimental group reflected individual treatment.

\section{Quality evaluation of the included literature}

The involved 24 articles were all randomized clinical controlled trials (13-36), but none explained whether their allocation was hidden, whether they were blinded, or whether there was withdrawal bias. Three articles did not explain whether their baseline data were comparable $(14,23,26)$, and the remaining 21 articles $(13,15-22,24,25$, 27-36) all indicated that the baseline data of the two groups were comparable; the reporting bias of these articles was unclear. The quality evaluation is shown in Table 2, and the risk of bias is shown in Figure 2.

\section{Results of the meta-analysis}

Meta-analysis of the rate of successfully treated cases A total of 4 articles reported the total rate of successfully treated cases, which was estimated using a fixed-effect model $\left(\mathrm{I}^{2}=0 \%\right)$. The results showed that the total rate of successfully treated cases following combined treatment of type $2 \mathrm{DN}$ with Bailing capsules based on the control group was 1.24 times (95\% CI: $1.11-1.38)$ that of the control group (Figure 3).

\section{Meta-analysis of the 24-h UTP level}

Nine articles reported changes in 24-h UTP before and 


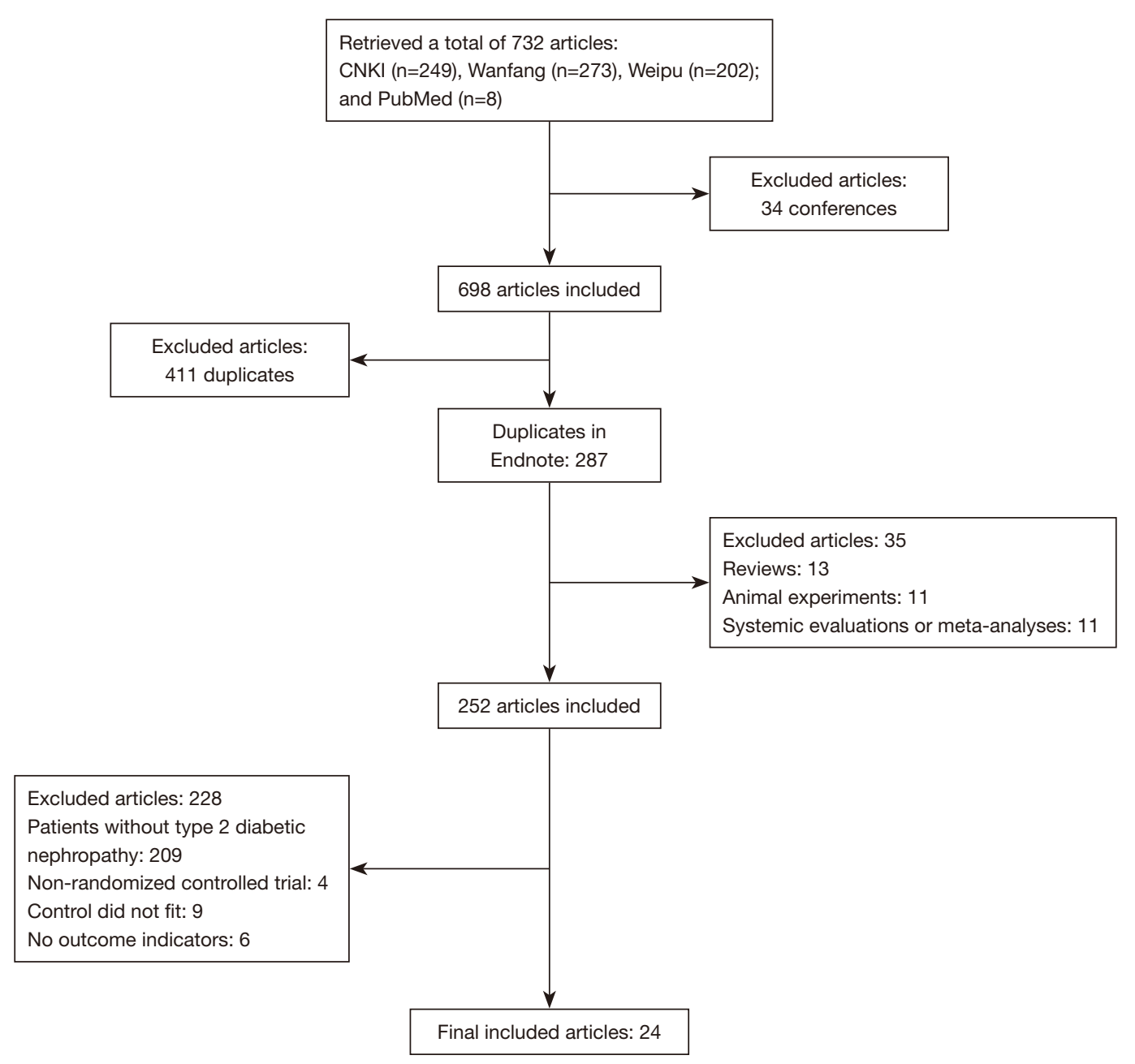

Figure 1 Retrieval flowchart of articles.

after treatment. The random-effects model $\left(\mathrm{I}^{2}=84 \%\right)$ was used to estimate the SMD. The difference in 24-h UTP before and after treatment with Bailing capsules based on the treatment of the control group for type $2 \mathrm{DN}$ was statistically significant, with an SMD of -0.61 (95\% CI: -1.01 to -0.22 ), as shown in Figure 4.

\section{Meta-analysis of UAER level}

Seven articles reported on changes in UAER level before and after treatment. The random-effects model $\left(\mathrm{I}^{2}=94 \%\right)$ was used to estimate SMD. Compared to the control group, the difference in UAER level before and after treatment of type $2 \mathrm{DN}$ with Bailing capsules based on the treatment of the control group was significantly lower, with an SMD of -1.56 (95\% CI: -2.34 to -0.78 ), as shown in Figure 5.

\section{Meta-analysis of Scr level}

There were 18 articles reporting on changes in Scr level before and after treatment. The random-effects model $\left(\mathrm{I}^{2}=88 \%\right)$ was used to estimate SMD. Compared to the control group, the difference in Scr level before and after treatment of type $2 \mathrm{DN}$ with Bailing capsules based on the treatment of the control group was significantly lower, with an SMD of 0.58 (95\% CI: -0.89 to -0.27 ), as shown in Figure 6.

\section{Meta-analysis of BUN}

Ten articles reported on changes in BUN level before and after treatment. The random-effects model $\left(\mathrm{I}^{2}=87 \%\right)$ was used to estimate SMD. Based on the treatment of the control group, the difference in BUN level before and after 


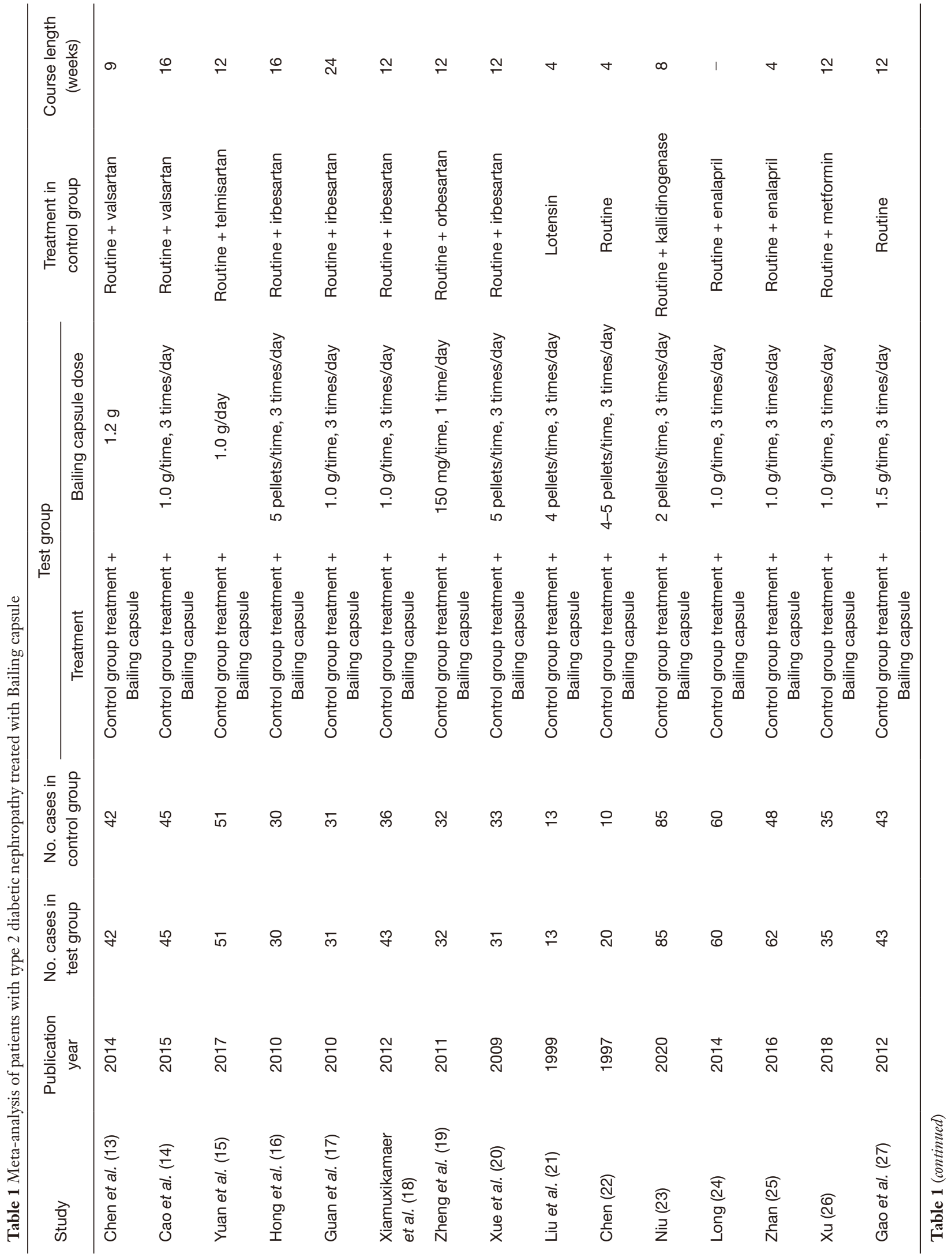




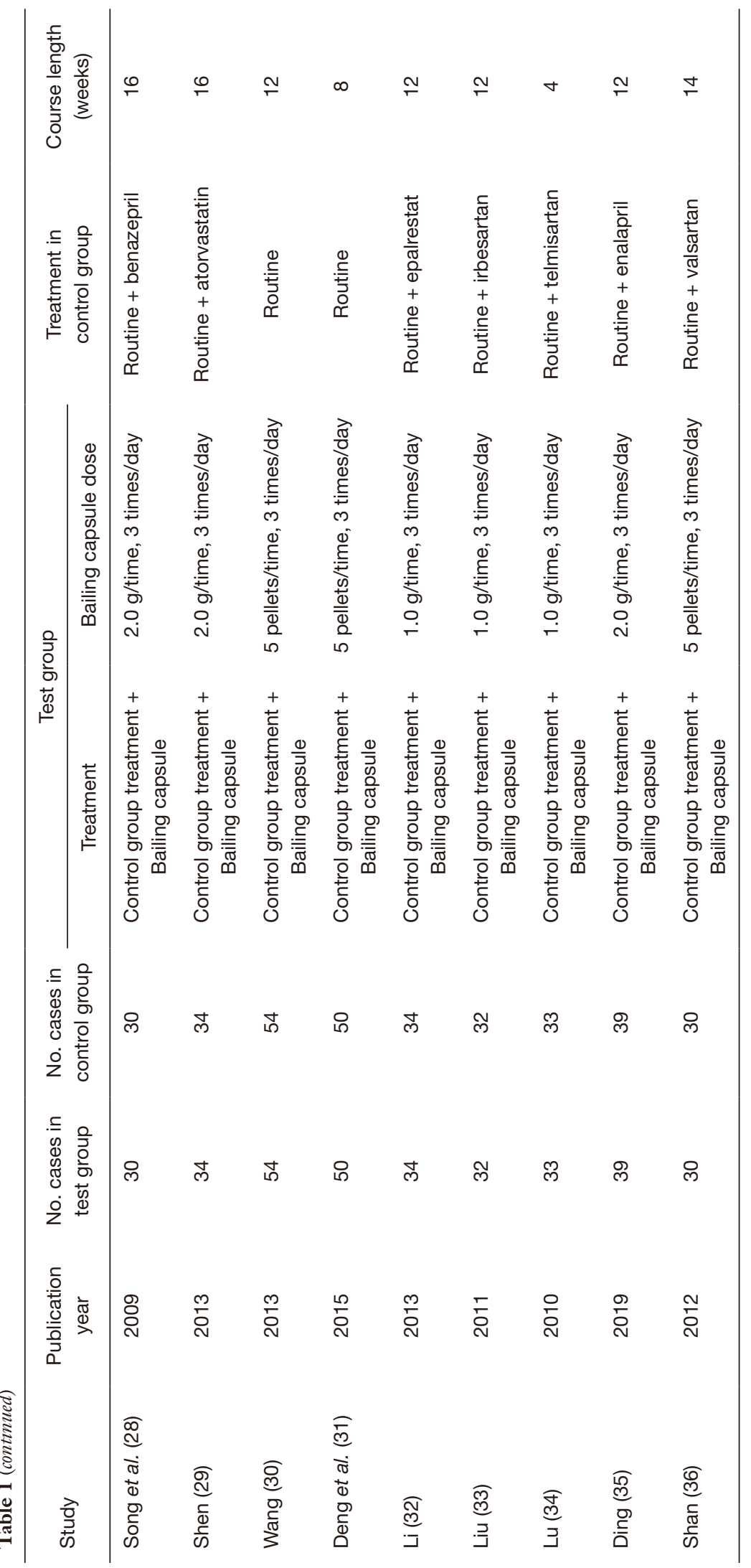


Table 2 Quality evaluation of the included literature patients with type 2 diabetic nephropathy treated with Bailing capsule

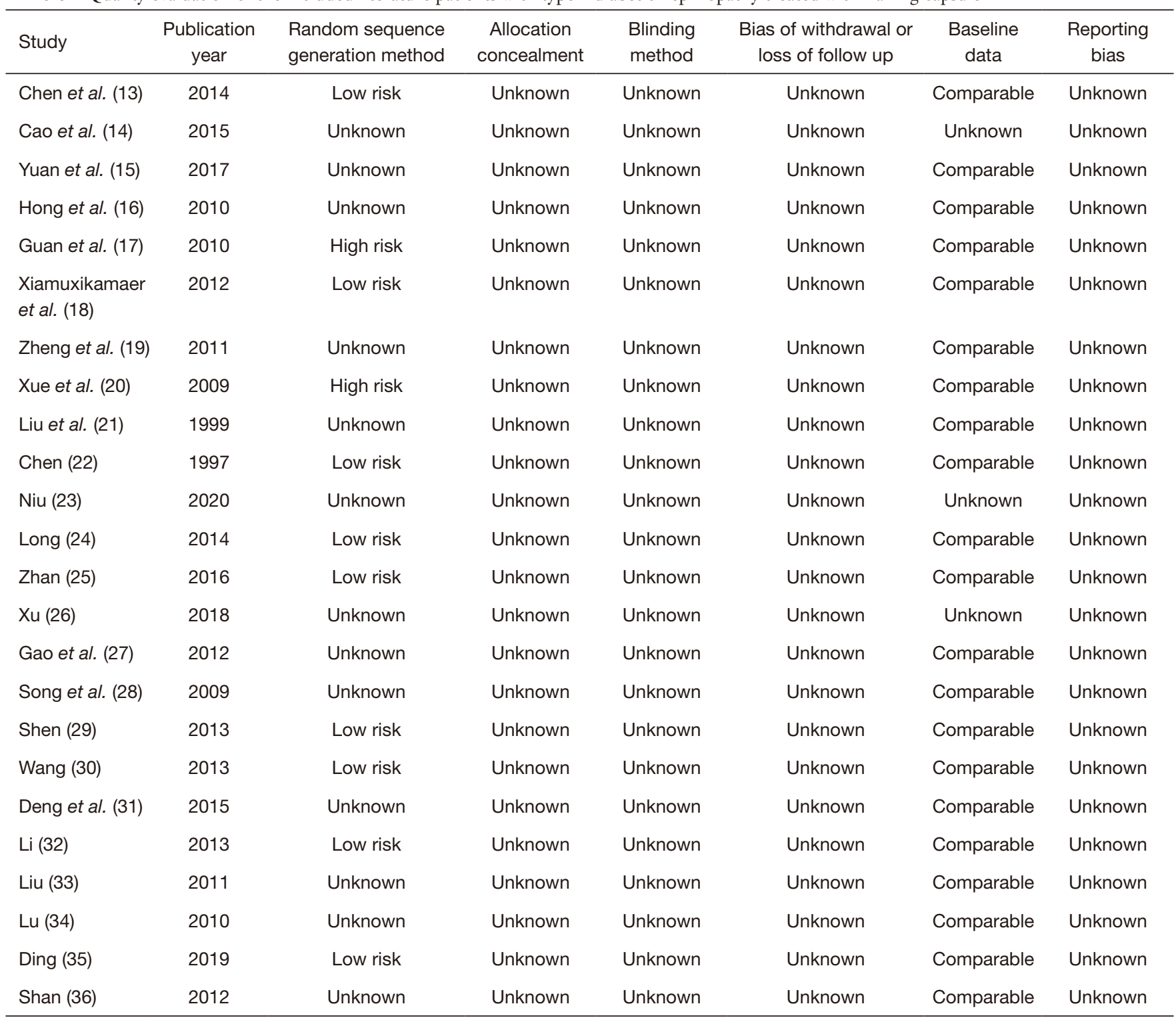

treatment of type $2 \mathrm{DN}$ with Bailing capsules based on the treatment of the control group was significantly lower, with an SMD of -0.73 before and after treatment (95\% CI: -1.16 to -0.29), as shown in Figure 7.

\section{Meta-analysis of serum potassium}

Two articles reported on changes in serum potassium before and after treatment. The fixed-effect model $\left(\mathrm{I}^{2}=0 \%\right)$ was used for SMD estimation. Based on the treatment of the control group, there was no statistically significant difference in serum potassium level before and after treatment combined with Bailing capsules for type $2 \mathrm{DN}$, with an SMD of -0.08 (95\% CI: $-0.37-0.20)$, as shown in Figure 8.

\section{Occurrence of adverse reactions}

Two articles reported the occurrence of adverse reactions in the two groups, and noted that there were no adverse reactions, such as liver and kidney dysfunction $(23,29)$.

Sensitivity analysis and publication bias evaluation Results of the sensitivity analysis did not show significant 


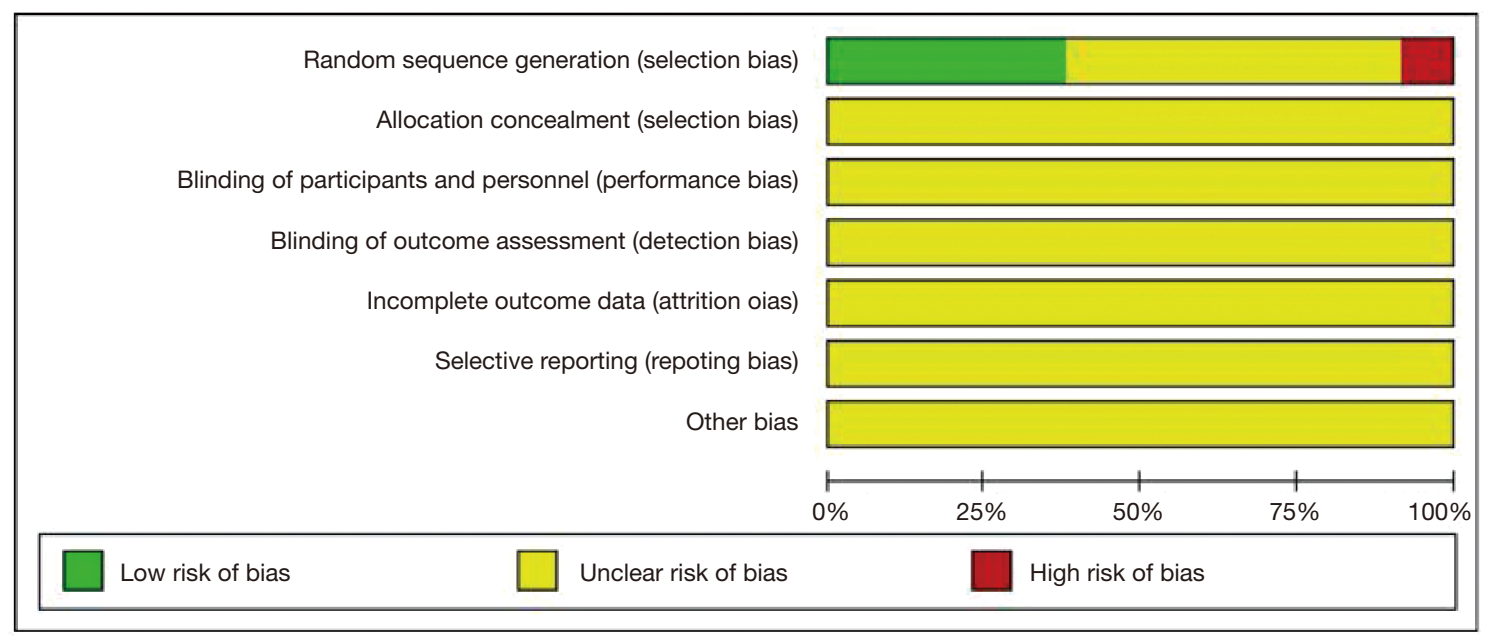

Figure 2 Quality bias evaluation of the included articles.

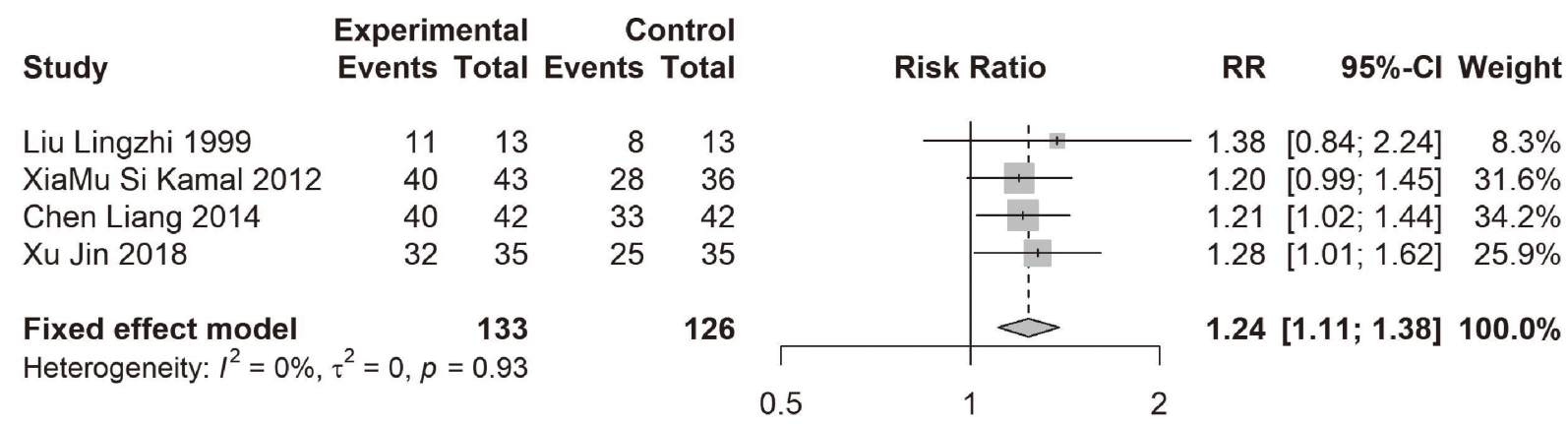

Figure 3 Forest map of meta-analysis of the total rate of successfully treated cases following routine treatment combined with Bailing capsule for type 2 diabetic nephropathy treatment. CI, confidence interval.

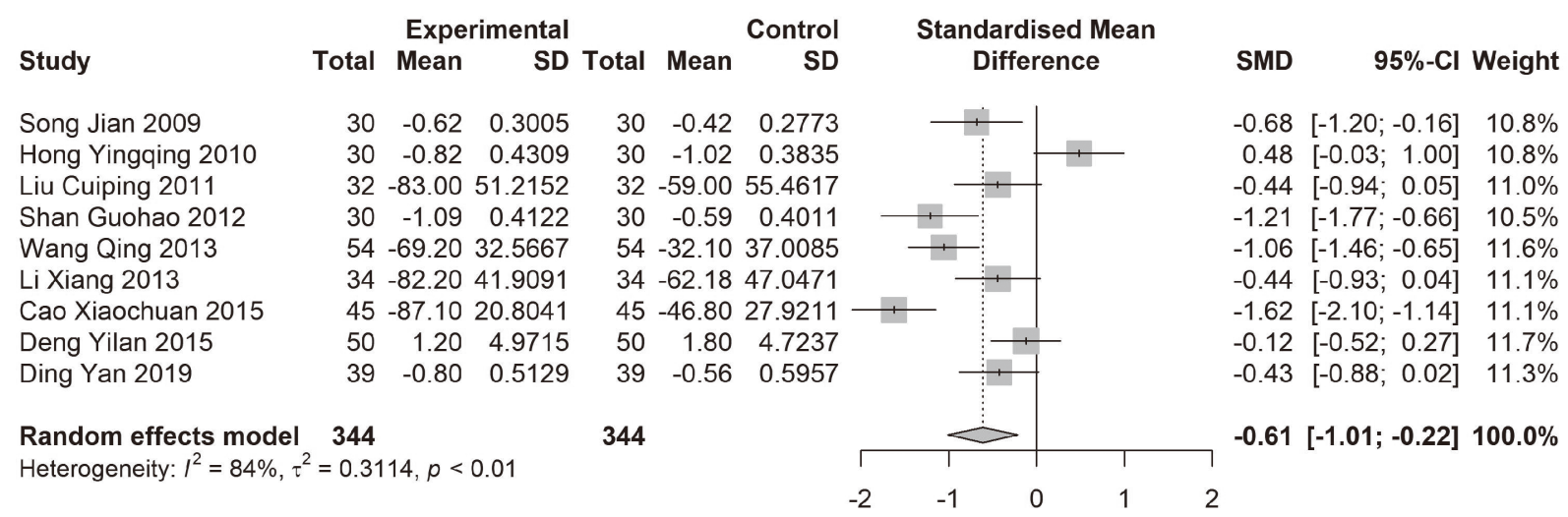

Figure 4 Forest map of meta-analysis of 24-h urinary total protein difference before and after treatment following routine treatment combined with Bailing capsule for type 2 diabetic nephropathy patients. CI, confidence interval; SD, standard deviation; SMD, standardized mean difference. 


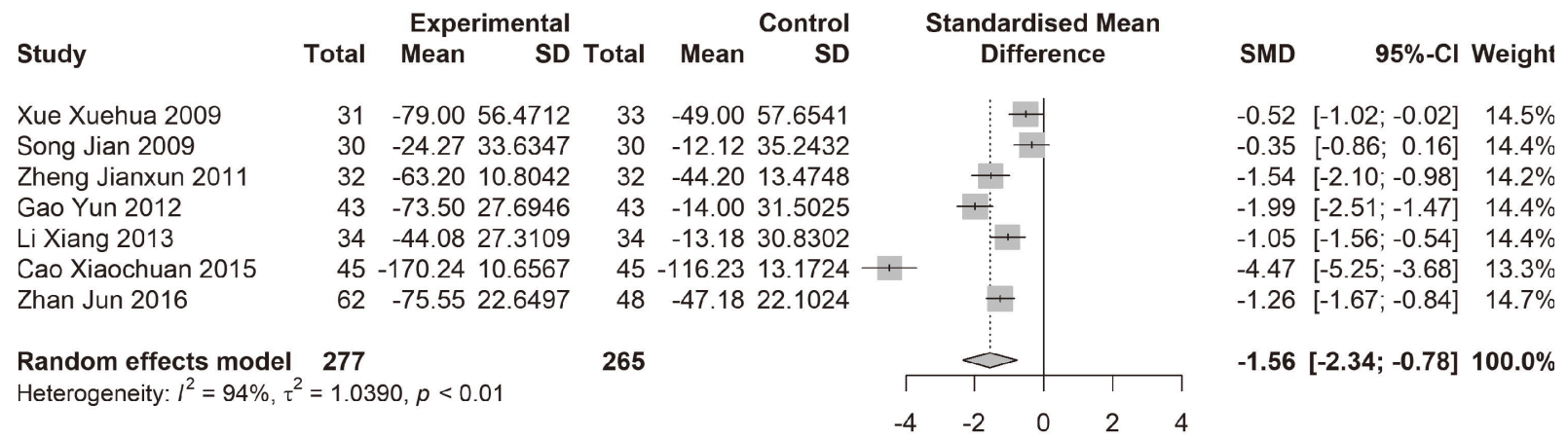

Figure 5 Forest map of meta-analysis of urine albumin excretion rate difference before and after treatment following routine treatment combined with Bailing capsule for type 2 diabetic nephropathy patients. CI, confidence interval; SD, standard deviation; SMD, standardized mean difference.

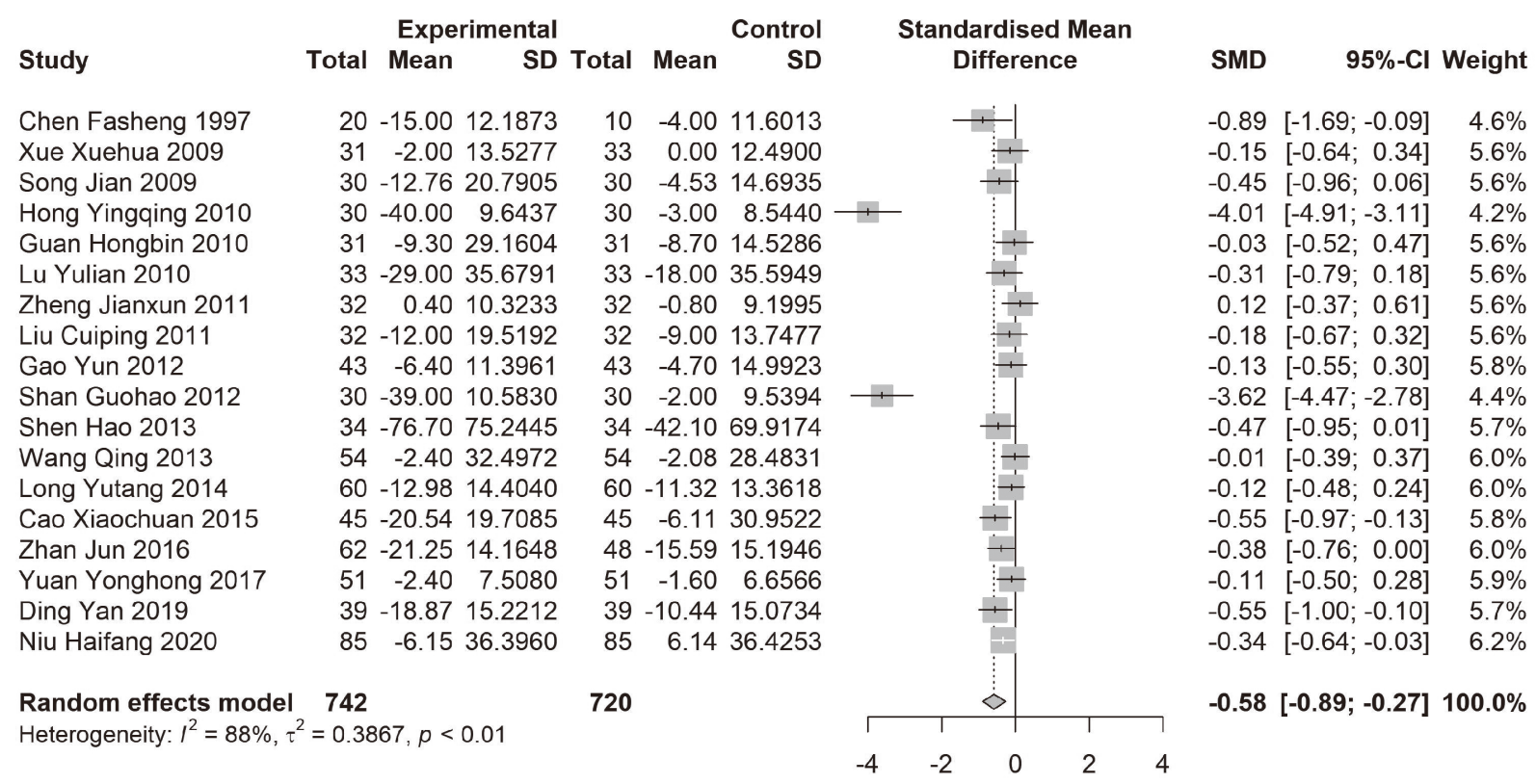

Figure 6 Forest map of meta-analysis of serum creatinine before and after treatment following routine treatment combined with Bailing capsule for type 2 diabetic nephropathy patients. CI, confidence interval; SD, standard deviation; SMD, standardized mean difference.

changes in the results of the study, indicating the results were stable. Because the meta-analysis of the rate of successful treatment and serum potassium included limited articles, no publication bias evaluation was performed. The remaining indicators (24-h UTP, UAER, Scr, and BUN levels) were analyzed using Egger's regression and Begg's rank correlation analysis; no publication bias of these indicators was found $(\mathrm{P}>0.05)$ (Table 3).

\section{Discussion}

$\mathrm{DN}$ is the main cause of ESRF, which is regarded as a global health concern. Studies have shown that the occurrence and development of DN can be controlled with strict control of blood sugar, blood pressure, and blood lipids; however, a fundamental therapeutic effect is still unachievable. Studies have shown that the use of some drugs for the long-term control of blood glucose level, angiotensin-converting 


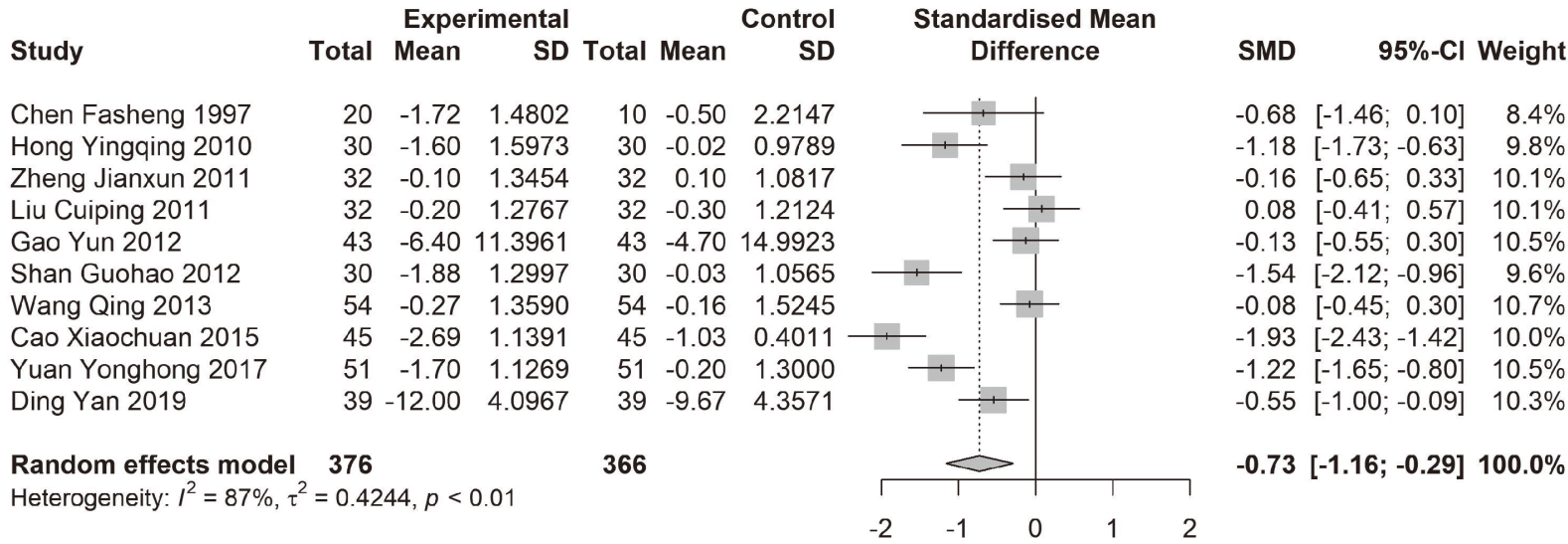

Figure 7 Forest map of meta-analysis of blood urea nitrogen before and after treatment following routine treatment combined with Bailing capsule for type 2 diabetic nephropathy patients. CI, confidence interval; SD, standard deviation; SMD, standardized mean difference.

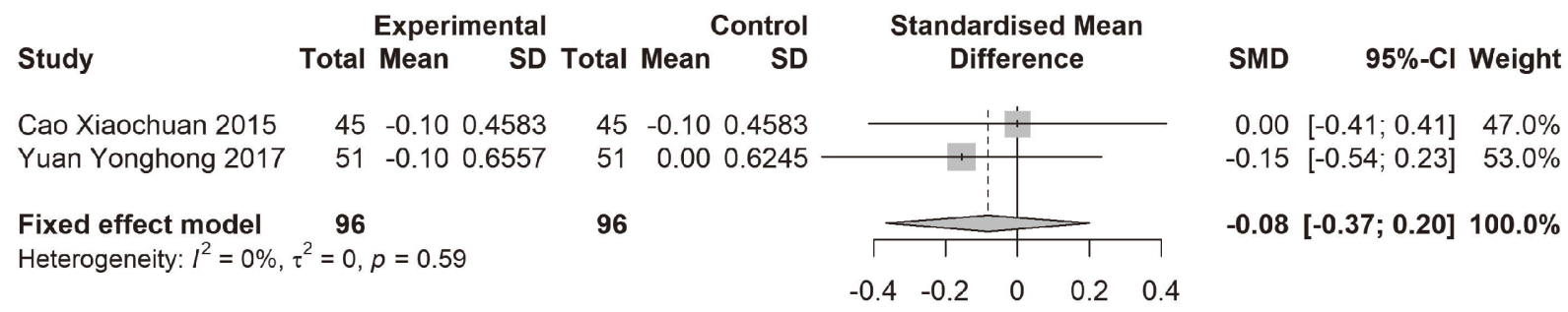

Figure 8 Forest map of meta-analysis of serum potassium before and after treatment following routine treatment combined with Bailing capsule for type 2 diabetic nephropathy patients. CI, confidence interval; SD, standard deviation; SMD, standardized mean difference.

Table 3 Evaluation of meta-analysis bias of routine treatment combined with Bailing capsule for type 2 diabetic nephropathy treatment

\begin{tabular}{|c|c|c|c|c|}
\hline \multirow{2}{*}{ Indicators } & \multicolumn{2}{|c|}{ Egger's regression } & \multicolumn{2}{|c|}{ Begg's rank correlation } \\
\hline & $\mathrm{t}$ & $P$ value & Z & $P$ value \\
\hline $\begin{array}{l}\text { 24-h urinary total } \\
\text { protein }\end{array}$ & -0.200 & 0.847 & -0.626 & 0.532 \\
\hline $\begin{array}{l}\text { Urine albumin } \\
\text { excretion rate }\end{array}$ & -2.323 & 0.068 & -1.352 & 0.176 \\
\hline Serum creatinine & -3.926 & 0.001 & -1.932 & 0.053 \\
\hline
\end{tabular}

enzyme inhibitors, or angiotensin receptor antagonists for $\mathrm{DN}$ can reduce the proteinuria of $\mathrm{DN}$ and protect kidney function; however, long-term follow-up results have demonstrated adverse effects, such as increased serum potassium and continued progress of kidney disease. DN belongs to the categories of edema, Xiaoke, and Guange in traditional Chinese medicine. It mainly causes the inability to solidify the essence and the deficiency of both qi and yin, and leads to blood stasis due to long-term disease; therefore, the root of treating the disease is to benefit qi, nourish yin, and keep the energy of the body (37).

Cordyceps sinensis is a Chinese medicine with a variety of biologic activities. Ben Cao Cong Xin states that the effects of Cordyceps sinensis are "protecting the lungs, nourishing the kidneys, stopping bleeding and resolving phlegm" (38). Bailing capsule is a medicinal preparation of Cordyceps sinensis that is made by low-temperature fermentation. It contains the same ingredients as the natural Cordyceps fungus and many potential biologically active ingredients, such as polysaccharides, adenosine, cordycepin, cordycepic acid, and ergosterol. Bailing capsule is reported to regulate hormone levels, increase blood circulation, lower blood pressure, inhibit platelet aggregation, as well as having anti-inflammatory and anti-anoxic effects; therefore, has protective effects on the kidney, lung, liver, as well as organs $(39,40)$.

In the present study, the results of the meta-analysis 
showed that the clinical efficacy of the test group was significantly better than that of the control group; the rate of successful treatment was 1.24 times that of the control group (95\% CI: 1.11-1.38); 24-h UTP, UAER, Scr, and BUN in the test group decreased significantly after treatment, and the difference before and after treatment was significantly lower than that of the control group, indicating that the clinical treatment method of adding Bailing capsule can significantly improve the renal function of type $2 \mathrm{DN}$. UTP and UAER levels are key indicators that reflect the degree of renal damage in $\mathrm{DN}$, and are independent risk factors for patients with DN to progress to ESRF. Reducing UTP and UAER can slow down the attenuation of the glomerular filtration rate and delay the progression of $\mathrm{DN}$, which is an effective treatment to protect the kidneys (41). The adenosine in Bailing capsules can scavenge free radicals, reduce lipid peroxides, increase superoxide dismutase content, improve renal blood flow, inhibit platelet aggregation, stabilize lysosomal membranes, and promote renal cell repair, thereby improving microcirculation, blocking and reducing renal microvascular disease, reducing urine albumin excretion, and improving renal function. Bailing capsules can also delay glomerular sclerosis by inhibiting the proliferation of glomerular mesangial cells under high glucose conditions (5). Bailing capsules have a male hormone-like effect, which is conducive to protein synthesis, thereby depleting blood nitrogen and reducing BUN level (42). The occurrence of DN has been found to be often accompanied by an increase in the expression of inflammatory factors, and the inflammatory response plays an important role in the occurrence and development of DN. Bailing capsules can reduce the production of inflammatory transmitters in routine treatment, thereby protecting the structure and function of renal tubular cell membranes, reducing damage to the kidney parenchyma, decreasing proteinuria level, and improving renal function.

In the present study, we found that the difference in serum potassium levels before and after treatment was not significantly different between the two groups, and it was reported that there was no liver and kidney dysfunction in the two groups in all of the articles. Although only two articles described this phenomenon, we believe that the combination of Bailing capsules with the first-line drugs of type $2 \mathrm{DN}$ is safe in clinical settings. Early systematic reviews on early DN have suggested that Bailing capsules combined with routine treatment is safe for early DN patients, but the sample sizes for clinical safety were small. Therefore, the safety of Bailing capsules in the treatment of type $2 \mathrm{DN}$ still needs to be verified by large-sample research and multicenter clinical trials.

The sensitivity meta-analysis found that the results of the rate of successfully treated cases and 24-h UTP, UAER, BUN, Scr, and serum potassium levels were relatively stable, with no study finding a significant impact on the research results. Because there were only a few articles on the rate of successfully treated cases and serum potassium levels, publication bias evaluation was not performed. The evaluation of publication bias for the remaining indicators (24-h UTP, UAER, Scr, and BUN levels) were analyzed by Egger's regression and Begg's rank correlation analysis, and no publication bias was found.

With the exception of low heterogeneity for the analysis of the rate of successfully treated case, the meta-analysis of the other indicators showed greater heterogeneity, which may be due to the following reasons: (I) the treatment measures between different studies are inconsistent; for example, in some studies, the control group underwent routine treatment combined with telmisartan, whereas in other studies only routine treatment was used in the control group; (II) the treatment courses of Bailing capsules are also different. Some studies had 9 weeks of treatment, whereas some had 16 weeks of treatment, and others had 24 weeks of treatment; (III) patient characteristics differ in studies; and (IV) the course of DN is inconsistent. These could be reasons for the high heterogeneity. A subgroup analysis based on the course of treatment was also performed. It was found that changes in the curative effect and renal function indexes were not associated with treatment time. The curative effect and renal function indexes were significantly better than those of the control group; however, heterogeneity was not significantly reduced.

This meta-analysis has the following limitations: (I) the quality of the clinical trial literature on traditional Chinese medicine preparation was poor; the studies did not indicate whether the allocation was hidden or blinded, whether there was withdrawal bias, and the reporting bias was unclear; (II) the heterogeneity of the research outcomes was high. As mentioned earlier, the different characteristics of the study participants in the different studies, and the different treatment schemes, doses, and treatment time, were all associated with the high heterogeneity; (III) the study sample sizes were generally small. There were also limited articles that reported on adverse reactions after treatment $(n=2)$. Therefore, the findings need to be further verified using a large sample in randomized clinical controlled trials. 


\section{Conclusions}

The meta-analysis found that the treatment of type 2 DN with routine therapy plus Bailing capsules was more effective than routine therapy alone, which can effectively improve renal function and significantly reduce levels of 24-h UTP/UAER, Scr, and BUN. Adverse reactions also decreased following routine treatment plus Bailing capsules. However, as the quality of included literature was low and the sample size was small, randomized double-blinded clinical trials with large sample sizes need to be conducted, particularly for the safety of long-term drugs, to fully evaluate the short- and long-term effectiveness and safety of Bailing capsules.

\section{Acknowledgments}

Funding: None.

\section{Footnote}

Reporting Checklist: The authors have completed the PRISMA reporting checklist. Available at http://dx.doi. org/10.21037/apm-20-1799

Conflicts of Interest: All authors have completed the ICMJE uniform disclosure form (available at http://dx.doi. org/10.21037/apm-20-1799). The authors have no conflicts of interest to declare.

Ethical Statement: The authors are accountable for all aspects of the work in ensuring that questions related to the accuracy or integrity of any part of the work are appropriately investigated and resolved.

Open Access Statement: This is an Open Access article distributed in accordance with the Creative Commons Attribution-NonCommercial-NoDerivs 4.0 International License (CC BY-NC-ND 4.0), which permits the noncommercial replication and distribution of the article with the strict proviso that no changes or edits are made and the original work is properly cited (including links to both the formal publication through the relevant DOI and the license). See: https://creativecommons.org/licenses/by-nc-nd/4.0/.

\section{References}

1. Whiting DR, Guariguata L, Weil C, et al. IDF diabetes atlas: global estimates of the prevalence of diabetes for 2011 and 2030. Diabetes Res Clin Pract 2011;94:311-21.

2. Guidelines for the prevention and control of type 2 diabetes in China (2017 Edition). Chinese Journal of Practical Internal Medicine 2018;38:292-344.

3. Umanath K, Lewis JB. Update on diabetic nephropathy: core curriculum 2018. Am J Kidney Dis 2018;71:884-95.

4. Flyvbjerg A. The role of the complement system in diabetic nephropathy. Nat Rev Nephrol 2017;13:311-8.

5. Zhao XM, Zhang Y, He XH, et al. Chinese herbal medicine Shenzhuo Formula treatment in patients with macroalbuminuria secondary to diabetic kidney disease: study protocol for a randomized controlled trial. Trials 2018;19:200.

6. Liu QQ, Du Xin, Tan JC. Research progress of Chinese and Western medicine in diabetic nephropathy. Tianjin Journal of Traditional Chinese Medicine 2019;36:205-8.

7. Dong HY, Yang YY, Han F, et al. Effect of Bailing Capsule combined with Alprostadil on immune index and clinical effect of senile diabetic nephropathy patients. Practical Geriatrics 2019;33:285-7+290.

8. Yuan XM, Li HX, Gao XY. Effect of Bailing capsule on the patients with stable chronic obstructive pulmonary disease. Journal of Xinxiang Medical University 2018;35:42-4+49.

9. Zhang Y, Effects of bailing capsule combing with angiotensin receptor blocker in the treatment of diabetic kidney disease: a meta-analysis. Hubei University of Traditional Chinese Medicine 2018.

10. Huang YL, Huang GD, Cai LK, et al. Effect and Safety of Bailing Capsule Combined with RAAS Inhibitors in Treatment of Early Diabetic Nephropathy: A Systematic Review. Chinese Archives of Traditional Chinese Medicine 2019;37:1290-7.

11. Oh SW, Kim S, Na KY, et al. Clinical implications of pathologic diagnosis and classification for diabetic nephropathy. Diabetes Res Clin Pract 2012;97:418-24.

12. JPT HS G. Cochrane Handbook for Systematic Reviews of Interventions Version 5.1.0. The Cochrane Collaboration, 2011 (2012-03-30). Available online: http:// www.Cochrane-handbook.org

13. Chen L, Chen LC, Xu LW. Clinical observation of valsartan combined with Bailing Capsule in the treatment of early diabetic nephropathy. Medical Aesthetics and Cosmetology (Mid-Month) 2014;156-7.

14. Cao XC. Effect analysis of valsartan combined with corbrin capsule in the treatment of early-stage diabetic nephropathy. China Modern Medicine 2015;22:97-9,101. 
15. Yuan YH. The Clinical Curative Effect of Telmisartan Combined with Bailing Capsule for Early type II Diabetic Nephropathy (With 51 Cases). Aerospace Medicine 2017;28:270-2.

16. Hong YQ. Irbesartan combined with Bailing Capsule in the treatment of senile type 2 diabetic nephropathy. Chinese Journal of Misdiagnostics 2010;10:5857-8.

17. Guan HB, He KP, Huan WM, et al. Effect of Irbesartan with Bailing Capsules on Microalbuminuria-to-creatinine Ratio and High-sensitive C-reactive Protein in Patients with Early Diabetic Nephropathy Chinese General Practice 2010;13.

18. Xiamuxikamaer MMTM, Guo J, Gulixian RYM. The effect of Irbesartan and Bailing capsules in the treatment of type 2 diabetic nephropathy. Guide of China Medicine 2012;588-9.

19. Zheng JG. Clinical observation of irbesartan and Bailing capsules in the treatment of type 2 diabetic nephropathy. Chin Mod Med 2011;18:66-7.

20. Xue XH. Clinical observation of irbesartan and Bailing capsules in the treatment of type 2 diabetic nephropathy. Shanxi Medical Journal 2009;38:45-6.

21. Liu LZ, Wu T, Zhang J. The Clinical Effects of Baling Capsules in Treating Diabetic Nephropathy. Central Plains Medical Journal 1999;35.

22. Chen FS. Effects of bailing capsule on type 2 diabetic nephropathy. New Journal of Traditional Chinese Medicine 1997;32-3.

23. Niu HF. Effects of bailing capsule combined with pancreatic kininogenase on early-onset type 2 diabetic nephropathy III period. Journal of Medical Forum 2020;41:94-6+100.

24. Long YT. Effect of Bailing capsule combined with enalapril on C-reactive protein and insulin resistance index in early type 2 diabetic nephropathy. Chinese Journal of Ethnomedicine and Ethnopharmacy 2014;23:37-8.

25. Zhan J, Zhang WN, Feng JT, et al. Study on the Effect of Bailing Capsule Combined with Enalapril on the CRP, Homa IR and Prognosis of Patients with Early Type 2 Diabetic Nephropathy. Medicine Healthcare Apparatus 2016;23.

26. Xu J. Effect of Bailing Capsule combined with metformin on serum inflammatory factors in patients with early diabetic nephropathy. Chinese Journal of Integrated Traditional and Western Nephrology 2018;19.

27. Gao Y, Yue F, Xu J. Effect of Bailing Capsules Combined with Ligustrazine on Platelet Activation and Microalbuminuria in Patients with Early Diabetic
Nephropathy. China Pharmacist 2012;15:233-4.

28. Song J, Li YH, Yang XD, et al. Effect of Combined Therapy with Bailing Capsule and Benazepril on Urinary Albumin Excretion Rate and C-reactive Protein in Patients with Early Diabetic Nephropathy. Chinese Journal of Integrated Traditional and Western Medicine 2009;29:791-3.

29. Shen H. Effect of atorvastatin combined with Bailin capsule on microinflammatory state of diabetic nephropathy. Journal of Clinical Internal Medicine 2013.

30. Wang Q. Effects of Bailing Capsule on the Cellular Immune Function of Type 2 Diabetic Nephropathy Patients. Chinese Journal of Arteriosclerosis 2013;21:1124-6.

31. Deng YL, Yin YS, Wei JZ, et al. Effect of Bailing Capsule on Ankle Brachial Index, Toe Brachial Index and Oxidative Stress in Diabetic Nephropathy with Normoalbuminuria. China Journal of Experimental Traditional Medical Formulae 2015;21:196-9.

32. Li X. Clinical Effect of Bailing Capsules Combined with Epalrestat Capsules in the Treatment of Early Diabetic Nephropathy. China Pharmacist 2013.

33. Liu CP. Effect of Irbesartan combined with Belling capsule on urinary albumin excretion rate and $\mathrm{C}$-reactive protein in patients with early diabetic nephropathy. Hebei Medical Journal 2011;33.

34. Lu YL. Effect of telmisartan combined with Bailing capsule on the treatment of diabetic nephropathy. Chinese Journal of Clinical Rational Drug Use 2010;3:43-4.

35. Ding Y. Effects of Enalapril combined with Bailing capsule on renal function and insulin resistance index in type 2 diabetic nephropathy patients. Chinese Journal of Convalescent Medicine 2019.

36. Shan GH. Effect of Bailing capsule on early diabetic nephropathy. Chinese Journal of Clinical Rational Drug Use 2012;05:81-2.

37. Lin HR, Mai ZH, Liang GQ, et al. Bailing Capsule Combined with Nifedipine Zyban Treating Patients with Hypertension Complicated with Chronic Renal Insufficiency and Its Influence on Cystatin C and 24h urine Protein Quantitative Level. Sichuan Medical Journal 2015;36:869-72.

38. Zhou F, Zhang M, Li LZ, et al. Effects of cordyceps sinensis extract on VEGF and renal function after experimental renal ischemia/reperfusion in rats. Zhejiang Clinical Medical Journal 2015;17:1678-80.

39. Jiang WF. Clinical and experimental study on the prevention and treatment of primary renal syndrome 
recurrence by Bailing Capsule. Guangzhou University of Chinese Medicine 2001.

40. Liu, XL, Wu YM. Progression of the mechanism of Cordyceps sinensis in the treatment of diabetic nephropathy. Shandong Medical Journal 2015;55:98-100.

41. Pilemann-Lyberg S, Lindhardt M, Persson F, et al. Serum uric acid and progression of diabetic nephropathy in type 1 diabetes. J Diabetes Complications 2018;32:470-3.

42. Chen YC, Chen YH, Pan BS, et al. Functional study of Cordyceps sinensis and cordycepin in male reproduction: A review. J Food Drug Anal 2017;25:197-205.

(English Language Editor: R. Scott)

Cite this article as: Sheng X, Dong Y, Cheng D, Wang N, Guo Y. Efficacy and safety of Bailing capsules in the treatment of type 2 diabetic nephropathy: a meta-analysis. Ann Palliat Med 2020;9(6):3885-3898. doi: 10.21037/apm-20-1799 